\title{
Progress in the Research of Virtual Trial Assembly
}

\author{
Yang $\mathrm{Li}^{2, \mathrm{a}}$, Jian $\mathrm{Yao}^{1,2, \mathrm{~b}^{*}}$ and Yuling Zhang ${ }^{1, \mathrm{c}}$ \\ ${ }^{1}$ College of Urban Construction, Zhejiang Shuren University, Zhejiang Hangzhou 310015, China \\ ${ }^{2}$ Institute of Structural Engineering, Zhejiang University, Zhejiang Hangzhou 310058, China \\ a308935674@qq.com, byaojian5803@qq.com, c1299522116@qq.com
}

\begin{abstract}
Keywords: Steel structure, Virtual trial assembly, Three-dimensional measurement techniques, Bolt hole, Procrustes analysis algorithm.

Abstract. There are two ways of trial assembly for steel structures which are well known as the physical trial assembly and the virtual trial assembly. The physical trial assembly is of high reliability but low economy. Nevertheless, the virtual trial assembly can save cost to the extreme extent and shorten the period of construction by comparison, which is especially true for the large and complex engineering under poor construction conditions. Summaries of virtual trial assembly are made in detail on its related engineering applications, 3D measurement techniques, algorithms of Procrustes Analysis used in China, Japan, Europe. An illustrative example for practical engineering is given in the end, which provides a reference for application and further research.
\end{abstract}

\section{Introduction}

The trial assembly for steel structure is usually required to ensure the successful installation on the construction site. There are two ways of trial assembly for steel structures: the physical trial assembly and virtual trial assembly. The physical trial assembly is a traditional and effective measure to control the quality and ensure the successful installation of component on construction site. However, the physical trial assembly needs space, equipment, enormous human and material resources and costs a lot. In recent years, with the rapid development of computer technology, the virtual trial assembly becomes a new method of trial assembly began to be concerned. It is a new technology that uses the computer and related technology to simulate the process of physical trial assembly in the CAD environment. As a result, the process of physical trial assembly is conserved, with many advantages as no need of the support of site, and saving the cost of trial assembly greatly, etc.

\section{Application situation of virtual trial assembly in China}

Application situation. In China, the virtual trial assembly method on physical trial assembly of engineering process is used by some scholars, engineers and technicians to improve the accuracy and efficiency of the process. Nevertheless, it is not widely used in the practical engineering and is lack of systematic research on the virtual trial assembly.

The application of virtual trial assembly technology is introduced by Li Yadong ${ }^{[1]}$ and Ding Yifeng et al. ${ }^{[2]}$ in some members of trusses in 2th area of Shanghai Tower and truss in third layer of the belt. The principle can be attributed to the following two points:

(1) The coordinate system for single fabricated members is established, and coordinates of control ports (control points) by measuring is obtained. Then feature points of outer contour and locating points of bolt hole group as control points are chosen.

(2) A unified global coordinate system is established for a number of fabricated rational members, then the local coordinates of control points of single member are transformed to coordinates in global coordinate system, forming two interface surfaces in each interface. The control point coordinates of the two interface surfaces are analyzed, and it is determined whether they are match or not based on coordinates difference.

The steel color stripe system is adopted in the bearing structure of Kunming New Airport Terminal which is composed of 7 color stripes and steel cantilever columns. The stripes are divided into 478 members with a total weight of 16,600 tons. They are special bending and torsion members which 
require precise size of interface, manufacturing and trial assembly. As a result, the virtual trial assembly technology is applied which is successful in practice ${ }^{[3]}$.

The virtual trial assembly technology is also introduced by Min Lan et al. in Turkey ATLAS $(1+1) \times 600 \mathrm{MW}$ supercritical coal-fired power station. This technology reduces the site occupied, saves a lot of manpower and material resources, improves efficiency in the steel plant and finally ensures the quality of steel members.

The main steps and their problems. The research and application of virtual trial assembly are still in its infancy in China and its application is only limited to a single project. The basic main steps of virtual trial assembly are substantially similar: Firstly, a theoretical model is established based on the design drawings. Secondly, the control points of actual members are measured to establish actual model. Then the theoretical model with actual models is compared in the local coordinate system, and the manufacturing precision and modification are checked. Finally, the actual models are assembled in a simulation environment, matching actual models with theoretical models in global coordinate system. The misalignment values between actual models and theoretical models are counted, so it is determined whether the assembly can be finished and the members need modification or not.

This virtual trial assembly method mainly has the following disadvantages.

(1) The total station for measurement is used, and consequently the accuracy, speed and degree of automation are limited. When the member is complex, the station is required for repeated conversion to measure all the feature points, inducing the complexity of the operation and measurement error.

(2) The engineering design production in china now mainly is 2D CAD drawings. However, virtual trial assembly needs 3D model data of structures. So the manufacturer needs to establish 3D model by their own which increased workload of virtual trial assembly.

(3) For unavoidable fabrication error, measured feature points of member generally can not correspond exactly with the theoretical value, so a solution should be proposed that can fit actual coordinates to theoretical coordinates and obtain the misalignment values. There is no relative research literature so far in China.

(4) There are thousands of members and millions of feature points in one project. If the coordinate transformation and the match is processed by manual operation, the workload is too much to be completed. The engineering applications described above are all manually operated.

(5) The error evaluation is simple, and there is also no visual display for virtual trial assembly.

(6) Now the data of measurement is simply entered into computer manually and there is no special software and supporting measurement methods. There are just some simple comparison of actual values with theoretical values and lack of coordinate transformation and systematic error analysis method.

\section{Study situation in Japan}

In Japan, the design and most of manufacture stages have been digitized and automated in steel bridge industry. Several computer systems have been developed by Japanese bridge companies ${ }^{[5,6]}$, which includes the following four main parts:

(1) Three-dimensional computer aided drawing system. This system is mainly used to read design drawings and then generate virtual component model to provide production numerical control (NC) data and manufacturing documents.

(2) Computer aided manufacturing system. This system is mainly used to provide NC data to CNC Equipment for CNC scribing, cutting, drilling, plate assembly, welding and ultrasonic testing and other steps.

(3) Virtual trial assembly. This system is mainly used to simulate trial assembly in computer environment.

(4) Production information management system. This system is mainly used to control the manufacture and management of production schedule. The following Content will highlight virtual trial assembly system. 
The system is developed by Yokogawa bridge company and Nagaoka University of Technology and called CATS system. It consists of two subsystems, 3D measurement system and simulation system.

The three-dimensional measurement system measures the configuration and dimension of members of the bridge structure. This system has been developed based on stereo-vision method using CCD cameras. For measuring long members, this system uses four CCD cameras and adopts the theory of photographic surveying system as shown in Fig.1. The long members such as main girders and stringers are carried straight by traverse cars into the visual field of CCD cameras and measured at the same time. Then, the travelling distance detector measures the straight travelling distance of each member. The vertical and horizontal displacements depending on small deformation of traverse railways are also measured by the laser optical system. Finally, the whole configuration of the member is obtained by linking every measured value, which is measured by CCD cameras and all the other measuring instruments.

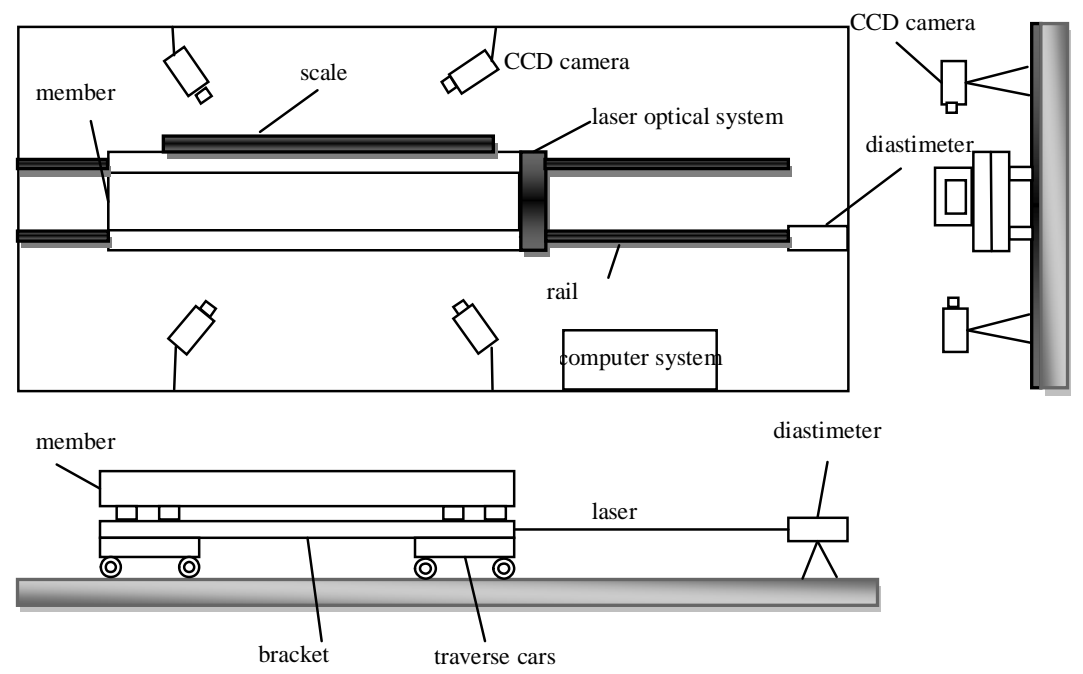

Fig.1 Three-dimensional measurement system

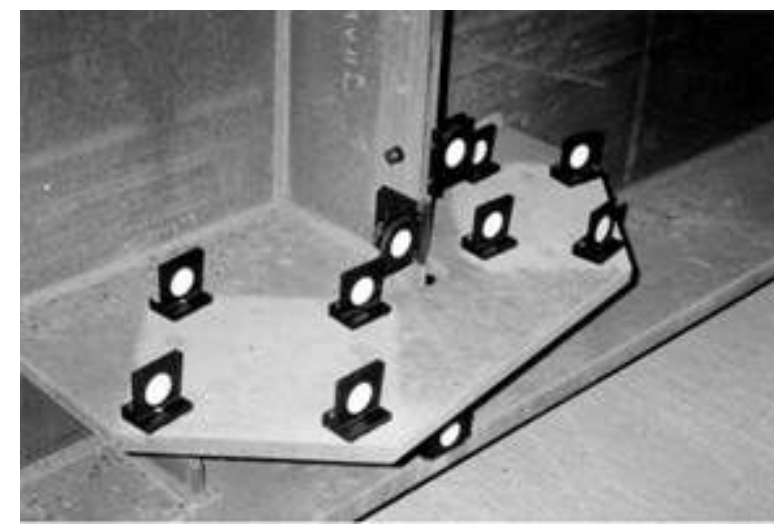

Fig. 2 Target placed on the bolt holes ${ }^{[5]}$

The measuring points are bolt holes for the field joint at the upper and lower flange. The targets shown in Fig. 2 are set on the measured points and their centers of circle pattern are three-dimensionally measured by CCD cameras.

This system simulates the process of shop assembly of a bridge on the basis of two kinds of data. One is data obtained by the preceding 3D measurement system and the other is design data given beforehand by the 3D CAD system Jupiter.

The data are compared with each other so as to ensure that each member is correctly fabricated in accordance with the designated sizes. If the difference in their data is larger than the acceptance criteria, the member is judged as disqualified and bad portions are corrected. When the whole members are correctly fabricated, they are brought into next procedure called "member assembly". In 
this simulation, each member is virtually placed so that bolt holes match to the proper holes of splice plates. After all members are completely placed, the final configuration of the bridge is checked. If the results of the check do not satisfy related restricts, configuration data of the structure such as camber, gap of field joint and distance of adjacent girders, are adjusted. This procedure is repeated until all control values above are satisfied.

\section{Study situation in Europe}

Principle. The match of bolt hole position in virtual trial assembly has been studied by Case et al. ${ }^{[8]}$ and Extended Orthogonal Procrustes Analysis (EOPA) and Generalized Procrustes Analysis (GPA) ${ }^{[8-11]}$ are proposed, both of which are based on Procrustes analysis.

(1) EOPA (Extended Orthogonal Procrustes Analysis)

There are two matrices A, B containing p points, which represents the actual coordinates and theoretical coordinate respectively. In order to achieve the match, the actual coordinates (A) should be translated and rotated to the greatest extent corresponding to the theoretical coordinate (B).

Supposing,

$$
B=A T+j t^{\mathrm{T}}+\boldsymbol{E}
$$

where, $\boldsymbol{j}^{\mathrm{T}}=(1,1, \ldots, 1) ; \boldsymbol{A}, \boldsymbol{B}$ are two known $p \times q$ coordinates matrix, $p$ represents the number of the positioning bolt holes in matrix, $q$ representing the calculated dimension. $q=2$ represents the simulation is in 2-dimensional space, $q=3$ represents the simulation is in 3-dimensional space. Let $\boldsymbol{t}_{\mathrm{q} \times 1}$ be a translation vector, $\boldsymbol{T}_{\mathrm{q} \times \mathrm{q}}$ a rotation matrix. Furthermore, considering an optional diagonal weight matrix $W_{\mathrm{r}}$ that assigns a different weight to the points according to their accuracy.

By formula (1), the following equation can be obtained

$$
\boldsymbol{E}=\boldsymbol{B}-\boldsymbol{A T}-\boldsymbol{J} \boldsymbol{t}^{\mathrm{T}}
$$

The objective is to calculate transform parameters $T$ and $t$ so that the square of 2 norm of $E$ can be made smallest.

The unknown transform parameters $T$ and $t$ are solved by the minimum variance conditions:

$$
\operatorname{tr}\left[\left(\boldsymbol{B}-\boldsymbol{A} \boldsymbol{T}-\boldsymbol{j t}^{T}\right)^{T} \boldsymbol{W}_{\mathrm{r}}^{2}\left(\boldsymbol{B}-\boldsymbol{A} \boldsymbol{T}-\boldsymbol{j} \boldsymbol{t}^{\mathrm{T}}\right)\right]=\min
$$

if $\boldsymbol{W}_{\mathrm{r}}^{2}$ admits Cholesky decomposition ${ }^{[8]}$, that is $\boldsymbol{W}_{\mathrm{r}}^{2}=\boldsymbol{Q}^{\mathrm{T}} \boldsymbol{Q}$

where, $Q$ are Cholesky decomposition of $\boldsymbol{W}_{\mathrm{r}}^{2}$.

$$
\text { let } \boldsymbol{A}_{\mathrm{w}}=\boldsymbol{Q A}, \boldsymbol{B}_{\mathrm{w}}=\boldsymbol{Q B}, \boldsymbol{j}_{\mathrm{w}}=\boldsymbol{Q j}, \boldsymbol{I}_{\mathrm{w}}=\left(\boldsymbol{I}-\frac{\boldsymbol{j}_{\mathrm{w}} \boldsymbol{j}_{\mathrm{w}}^{\mathrm{T}}}{\boldsymbol{j}_{\mathrm{w}}^{\mathrm{T}} \boldsymbol{j}_{\mathrm{w}}}\right),
$$

matrix $\boldsymbol{A}_{\mathrm{w}}, \boldsymbol{B}_{\mathrm{w}}, \boldsymbol{I}_{\mathrm{w}}, \boldsymbol{j}_{\mathrm{w}}$ are $\boldsymbol{A}, \boldsymbol{B} 、 \boldsymbol{I}, \boldsymbol{j}$ which take weight factor into account.

By computing the spectral decomposition of matrix product: $\boldsymbol{A}_{\mathrm{w}}^{\mathrm{T}} \boldsymbol{I}_{\mathrm{w}} \boldsymbol{B}_{\mathrm{w}}=\boldsymbol{V} \boldsymbol{D} \boldsymbol{W}^{\mathrm{T}}$.

The transformation parameters can be directly determined from:

rotate matrix $\boldsymbol{T}=\boldsymbol{V} \boldsymbol{W}^{\mathrm{T}}$

translate vector $\boldsymbol{t}=\left(\boldsymbol{B}_{\mathrm{w}}-\boldsymbol{A}_{\mathrm{w}} \boldsymbol{T}\right)^{\mathrm{T}} \frac{\boldsymbol{j}_{\mathrm{w}}}{\boldsymbol{j}_{\mathrm{w}}^{\mathrm{T}} \boldsymbol{j}_{\mathrm{w}}}$

(2) GPA (Generalized Procrustes Analysis)

EOPA solves the problem of matching the two matrices (actual coordinates and theoretical coordinates of single member) in virtual trial assembly. EOPA can maximize the correspondence 
between actual coordinates and theoretical coordinates. But in some cases, we should maximize the correspondence between actual coordinates in different members. Therefore, another calculation method of virtual trial assembly, GPA, has been proposed. GPA can estimate transform parameters $T_{i}$ and $t_{i}$. These transform parameters can maximize correspondence between two or more actual coordinates matrixes towards the centroid coordinates matrixes. Table 1 shows the differences between EOPA and GPA. Name Statistics of reciprocal misalignments between the centers of the assembly bolt holes as reciprocal misalignments and Statistics of hole misalignments between the theory center and assembly center of the bolt hole as theoretical misalignments.

\begin{tabular}{lll}
\hline Calculation Method & EOPA & GPA \\
\hline theoretical misalignments & small & big \\
reciprocal misalignments & big & small \\
\hline
\end{tabular}

\section{Table 1 Comparison EOPA with GPA}

The GPA method is described in the following:

Let $\boldsymbol{A}_{1}, \ldots, \boldsymbol{A}_{\mathrm{m}}(\mathrm{m} \geq 2)$ be models of $m$ members which needs virtual trial assembly. Both of them are $p \times 3$ matrixes, each matrix includes coordinates of the same $p$ points, and in different reference systems. $Z$ is theoretical coordinates of the model of the structure.

$A_{\mathrm{i}}$ and $Z$ satisfy similar relationship like formula (1),that is

$$
\boldsymbol{Z}=\boldsymbol{A}_{i} \boldsymbol{T}_{i}+\boldsymbol{j t}_{i}^{\mathrm{T}}+\boldsymbol{E}_{i}
$$

Where, $\boldsymbol{E}_{i}$ is a random error matrix

Let

$$
\boldsymbol{A}_{i}^{\mathrm{pr}}=\boldsymbol{A}_{i} \boldsymbol{T}_{i}+\boldsymbol{j} \boldsymbol{t}_{i}^{\mathrm{T}}
$$

where $\boldsymbol{A}_{i}^{\mathrm{pr}}$ represents matrix $\boldsymbol{A}_{\mathrm{i}}$ after rotation and translation.

The objective is to obtain transform parameters $t_{\mathrm{i}}, T_{\mathrm{i}}(\mathrm{i}=1, \ldots, \mathrm{m})$ for each matrix.

Among the possible infinite solutions, the choice falls on the unique one satisfying the following objective function:

$$
S=\operatorname{tr} \sum_{i=1}^{m} \sum_{k=i+1}^{m}\left\{\begin{array}{l}
{\left[\left(\boldsymbol{A}_{i} \boldsymbol{T}_{i}+\boldsymbol{j}_{i}\right)-\left(\boldsymbol{A}_{k} \boldsymbol{T}_{k}+\boldsymbol{j} \boldsymbol{t}_{k}\right)\right]^{\mathrm{T}}} \\
\times\left[\left(\boldsymbol{A}_{i} \boldsymbol{T}_{i}+\boldsymbol{j} \boldsymbol{t}_{i}\right)-\left(\boldsymbol{A}_{k} \boldsymbol{T}_{k}+\boldsymbol{j} \boldsymbol{t}_{k}\right)\right]
\end{array}\right\}=\min
$$

that is

$$
S=\sum_{i=1}^{m} \sum_{k=i+1}^{m}\left\|\boldsymbol{A}_{i}^{\mathrm{pr}}-\boldsymbol{A}_{k}^{\mathrm{pr}}\right\|=\min
$$

where $S$ represents misalignments between the centers of the assembly bolt holes.

One of the methods for the problem solution is based on the definition of matrix $\mathrm{C}$ which is centric of the set of matrices ${ }^{[8]}$.

$$
\boldsymbol{C}=\frac{1}{\mathrm{~m}} \sum_{i=1}^{\mathrm{m}} \boldsymbol{A}_{i}^{\mathrm{pr}}
$$

This method does not directly find out the solutions for $c_{\mathrm{i}}, t_{\mathrm{i}}$ and $T_{\mathrm{i}}$ that minimize Eq. (9), but makes use of the properties of the geometrical centroid, till an optimal approximation is obtained for it, satisfying the upon mentioned minimum function. In practice, the method consists of an iterative updating of the centroid till convergence, that is when the minimum condition is satisfied. It is 
proved $^{[12]}$ that the updated centroid corresponds to the least squares approximation of the real matrix $Z$. The following describes specific steps of the method:

1) Calculating centric matrix $\mathrm{C}$ and then matching $\boldsymbol{A}_{i}^{\mathrm{pr}}=\boldsymbol{A}_{i} \boldsymbol{T}_{i}+\boldsymbol{j} \boldsymbol{t}_{i}^{\mathrm{T}}$ and $\mathrm{C}$ is calculated by applying Eq.(10);

2) Matching new matrix of previous result $\boldsymbol{A}_{i}^{\mathrm{pr}}=\boldsymbol{A}_{i} \boldsymbol{T}_{i}+\boldsymbol{j} \boldsymbol{t}_{i}^{\mathrm{T}}, \boldsymbol{C}=\frac{1}{\mathrm{~m}} \sum_{i=1}^{\mathrm{m}} \boldsymbol{A}_{i}^{\mathrm{pr}}$ repeat this iterative process until the centric matrix $C$ is convergent, that is the amount of change in the centroid matrix less than a value $^{[8]}$. So we get the position of bolt holes after virtual trial assembly.

3) Then calculating the transform parameters $t_{i}, T_{i}$ by initial position and final position of bolt holes.

(3) An application example of GPA

Fig. 3 shows two steel plates with same dimensions, which join each other by 2, 4 bolt holes and join supports by 1, 3, 5, 6 bolt holes. The objective is to identify the best joining position, taking into account the actual dimensions and the mounting constraints that can differ from the nominal ones. Let $1,3,5,6$ be constraint points which claims the actual coordinates and theoretical coordinates to be as close as possible, 2, 4 be tie points which can relax the theoretical misalignments but claim to minimize reciprocal misalignments for connecting members. Each plate is a model which is a matrix defined by four points of centers of bolt holes in specific order. Coordinate system of these points can be arbitrary.
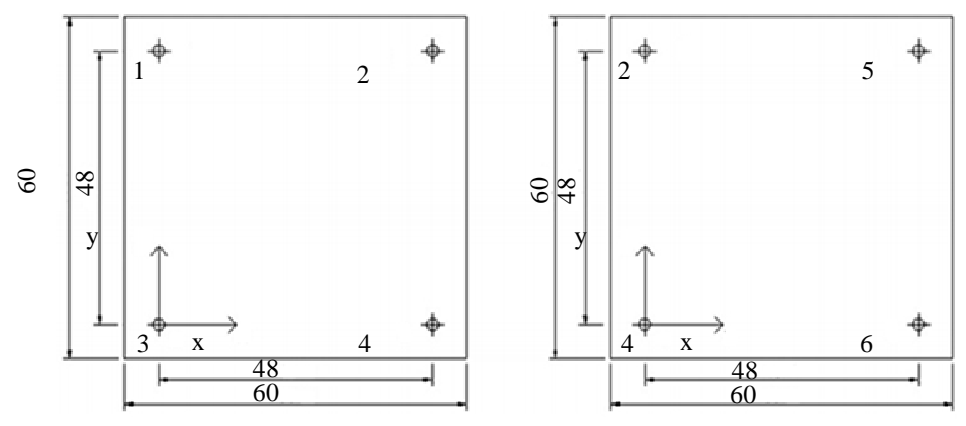

Fig.3 Two plate used for the example

By steps described before, we can get optimal connection, meet the minimum variance principles, and other constraints may be attached.

In the process of computing, the constraint points can be given a weighting factor greater than 1 in order to make the central point of the constraint points closer to the theoretical value. Finally, the coordinate matrix, centroid matrix, reciprocal misalignments and theoretical misalignments of initial and final model can be provided.

Table 2 shows the data of theoretical model required in virtual trial assembly. Each hole position includes two attributes: ID and theoretical coordinates value. ID is unique in one project, each bolt holes only has one unique ID to be Identification attributes. Coordinates can be 2D or 3D. All theoretical coordinates in one project must be in one coordinate system.

$x, y$ represent actual coordinates and $X, Y$ represent theoretical coordinates in table 2 and Table 3. 


\begin{tabular}{cccccc}
\hline & ID & $\mathrm{x}[\mathrm{cm}]$ & $\mathrm{y}[\mathrm{cm}]$ & $\mathrm{X}[\mathrm{cm}]$ & $\mathrm{Y}[\mathrm{cm}]$ \\
\hline \multirow{4}{*}{ Plate 1 } & 1 & 1.5 & 47.0 & 0.0 & 48.0 \\
& 2 & 48.5 & 49.0 & 48.0 & 48.0 \\
& 3 & 0.5 & 2.0 & 0.0 & 0.0 \\
& 4 & 49.0 & 0.5 & 48.0 & 0.0 \\
\hline \multirow{4}{*}{ Plate 2 } & 2 & 1.0 & 46.5 & 48.0 & 48.0 \\
& 5 & 48.0 & 47.0 & 96.0 & 48.0 \\
& 4 & 0.0 & 1.0 & 48.0 & 0.0 \\
\hline
\end{tabular}

Table 2 Measured coordinates of the points belonging to the two plates defined in two local independent reference system and design coordinates of the points in global coordinate system

\begin{tabular}{cccccc}
\hline & \multirow{2}{*}{ ID } & \multicolumn{2}{c}{ First assemble } & \multicolumn{2}{c}{ Second assemble } \\
\cline { 3 - 6 } & & $\mathrm{X}[\mathrm{cm}]$ & $\mathrm{Y}[\mathrm{cm}]$ & $\mathrm{X}[\mathrm{cm}]$ & $\mathrm{Y}[\mathrm{cm}]$ \\
\hline \multirow{4}{*}{ Plate 1} & 1 & 0.6250 & 46.3750 & 0.6250 & 46.3750 \\
& 2 & 47.6250 & 48.3750 & 47.6250 & 48.3750 \\
& 3 & -0.3750 & 1.3750 & -0.3750 & 1.3750 \\
& 4 & 48.1250 & -0.1250 & 48.1250 & -0.1250 \\
\hline \multirow{4}{*}{ Plate 2 } & 2 & 48.6854 & 46.5626 & 48.9660 & 47.1963 \\
& 5 & 95.6866 & 46.9366 & 95.9629 & 47.9335 \\
& 4 & 47.5634 & 1.0654 & 48.1957 & 1.6919 \\
& 6 & 96.0646 & 1.4354 & 96.6925 & 2.4366 \\
\hline
\end{tabular}

Table 3 Coordinates of the points in global coordinate system after virtual trial assembly

According to GPA described above, a Matlab program of virtual trial assembly is developed for trial assembly of plate 1 and plate 2 . Virtual trial assembly commissions twice, firstly the constraint points weighting factor is tried to make 1 , and secondly the 5 th constraint point weighting factor is made to be 50, finally the other constraint points weighting factor is 1 .Matching result is shown in Table 3, the specific process can be referred in literature ${ }^{[13]}$.

Table 3 shows the 5 th point of plate 2 weighting 50 which is much closer to the theoretical value than ones weighting 1.But,in the same time, other points of plate 2 will deviate from its theoretical value more. So, when the key point of trial assembly identified and given a large (appropriate) weights, and the other points is defined as obedience point, the virtual trial assembly can be completed reasonably.

Engineering Application. Case ${ }^{[8]}$ et al. have used GPA described above to simulate virtual trial assembly of New Safe Confinement of the Chernobyl nuclear reactor, and achieved good results. The New Safe Confinement of the nuclear reactor is an arched steel shell, with length $257 \mathrm{~m}$, width $162 \mathrm{~m}$, height $105 \mathrm{~m}$. The circle pipe is used in Steel members, and drilled holes are used in the end welded gusset plate. The high strength bolts for connecting are used in the structure. Node consists of a main steel plate and a number of secondary steel plates welded on the main steel plate, as is shown in Fig.4. Bolt diameter is $30 \mathrm{~mm}$, aperture is $33 \mathrm{~mm}$. Each bolt hole position tolerance is $3 \mathrm{~mm}$. 


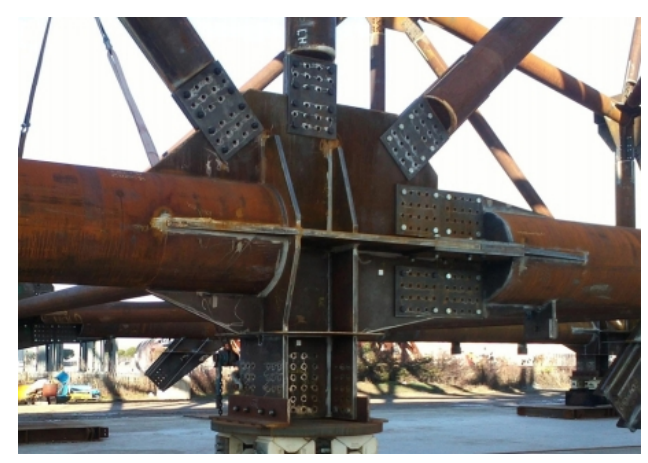

Fig.4 Example of joining among a node and the pipe elements

Three-layer reticulated dome of arch structure is used as an example, the following shows several simulations under different constraints.

The part of three-layer reticulated dome includes more than 3000 bolt holes, 281 members, and each member is represented by a matrix composed by 3D coordinates of some centers of positioning bolt holes. The temperature factors have been taken into account during the measurement of members. The result is shown in Table 4 and Table 5.

(1) Minimum constraint solution

The four outmost support nodes of the structure are chosen as constraint points and other nodes as tie points. The objective is to find matches satisfying the minimum variance principle. The result of minimum constraint solution shows that reciprocal misalignments of $98.93 \%$ bolt holes satisfy admitted tolerances in the project, but theoretical misalignments of $38.57 \%$ bolt holes are more than $3 \mathrm{~mm}$. Nevertheless, having fixed only 4 constraint points, this solution doesn't exactly respect some of the design coordinate values.

(2) Maximum constraint solution

Each nodes is constrained, so members can satisfy the design coordinate values as much as possible. In this constraint conditions, the result of maximum constraint solution shows that theoretical misalignments of $95.54 \%$ bolt holes satisfy admitted tolerances in the project, but reciprocal misalignments of $19.86 \%$ bolt holes are more than $3 \mathrm{~mm}$. The solution maximally satisfies the requirements of the design coordinate values, but it reduced the possibility of assembling. In order to be assembled, the $19.86 \%$ bolt holes requires a more detailed analysis. For example, a hole clearly exceeds the tolerance value of the case (but not beyond). In this case, simply move the splice plate, adjusting the relative positions of other holes, it may find that it can be fitted on. This requires a trial operation in the CAD environment.

\begin{tabular}{lcrlr}
\hline & \multicolumn{2}{l}{$\begin{array}{l}\text { minimum } \\
\text { constrained solution }\end{array}$} & \multicolumn{2}{l}{ all constrained } \\
solution & \\
\hline Total points & 1407 & & 1407 & \\
$\mathrm{R}<3 \mathrm{~mm}$ & 1392 & $98.93 \%$ & 1129 & $80.24 \%$ \\
$3 \mathrm{~mm}<\mathrm{R}<6 \mathrm{~mm}$ & 14 & $1.00 \%$ & 67 & $17.91 \%$ \\
$\mathrm{R}>6 \mathrm{~mm}$ & 1 & $0.07 \%$ & 11 & $1.85 \%$ \\
\hline
\end{tabular}

Table 4 Statistics of reciprocal misalignments for the minimum constrained solution and the all constrained solution ${ }^{[8]}$

\begin{tabular}{lcrcr}
\hline & $\begin{array}{l}\text { minimum } \\
\text { constrained solution }\end{array}$ & \multicolumn{2}{l}{$\begin{array}{l}\text { all constrained } \\
\text { solution }\end{array}$} \\
\hline Total points & 1407 & & 1407 & \\
$\mathrm{R}<3 \mathrm{~mm}$ & 1074 & $61.43 \%$ & 1671 & $95.54 \%$ \\
$3 \mathrm{~mm}<\mathrm{R}<6 \mathrm{~mm}$ & 571 & $32.63 \%$ & 67 & $3.83 \%$ \\
$\mathrm{R}>6 \mathrm{~mm}$ & 104 & $5.94 \%$ & 11 & $0.63 \%$ \\
\hline
\end{tabular}

Table 5 Statistics of theoretical misalignments for the minimum constrained solution and the all constrained solution ${ }^{[8]}$ 
Both research and applications show that the result of virtual trial assembly is good to meet expected objective. Using the steps proposed by the exact algorithm model, the results can be assembled in a very short period of time and do not depend on how much the number of models. So the solution of virtual trial assembly can be extended to other areas of complex engineering project such as complex architecture engineering.

\section{Conclusion}

Technology of virtual trial assembly in the international arena has been relatively perfect, while it is still in its infancy in China. To promote the application of this technology in the field of Chinese engineering, still we should make efforts in the following areas.

Use three-dimensional measurement techniques to measure the coordinates of bolt holes and size of members for improving efficiency and reducing errors.

By accelerating three-dimensional model of design production in the field of engineering design, automation management can be realized easily in the whole process of construction and manufacturing.

Algorithm of virtual trial assembly proposed by literature ${ }^{[8]}$ has good maneuverability and realizability,but specialized software for virtual trial assembly is required.

\section{Acknowledgements}

The authors are grateful for the financial support received from the Science and Technology Department of Zhejiang Province (Project No.: 2014C31006).

\section{References}

[1] Y.D.Li:J. CONSTRUCTION TECHNOLOGY, 2012, 41(18):23-26. ( in Chinese )

[2] Y.F.Ding,H.Lu and W.J.Li:J. Journal of Information Technology in Civil Engineering and Architecture, 2012, (1):52-56.

[3] L.Min,Y.Wang and J.Feng :J. Industrial construction, 2013. ( in Chinese )

[4] J.Y.Tang, L.N.Wu, and G.H.Liao:J. CONSTRUCTION TECHNOLOGY, 2009, 38(12):21-24. ( in Chinese )

[5] S.Tamai, Y.Yagata and T.Hosoya :J. Journal of Constructional Steel Research, 2002, 58(1): 151-192

[6] J.P.Li :J. Steel structure, 2013, 28:58-61. DOI:10.3969/j.issn.1007-9963.2013.04.014. ( in Chinese )

[7] L.Guo. Study on Principle and Implementation Method of 3D Precision Positioning in Large Field Working Space [D]. Tianjin University, 2008. DOI:10.7666/d.y1531209. ( in Chinese )

[8] F.Case, A.Beinat, F.Crosilla and I M.Alba :J. Aution in Construction, 2014, 37(1):155-165.

[9] P H.Schönemann, R M.Carroll :J. Psychometrika, 1969, 1969(2):i-19.

[10] P H.Schönemann :J. Psychometrika, 1966, 31:1-10. DOI:10.1007/BF02289451.

[11] J C.Gower :J. Psychometrika, 1975, 40(1):33-51.

[12] A J.Collins :J. Journal of Classification, 1992, 9:173-175. DOI:10.1007/BF02618483. 
[13] Y.Li. Methods Study of Virtual Trial Assembly of Steel Structure [D]. Zhejiang University , 2015. ( in Chinese ) 